\title{
Development of electromagnetic cascading in the Sun's magnetic field
}

\author{
A. Mahrous and N. Inoue \\ Department of Physics, Saitama University, Urawa 338, Japan \\ Received 23 July 2001 / Accepted 24 January 2002

\begin{abstract}
The study of particle cascading initiated by Extremely High Energy (EHE) photons in the Sun's magnetic field offers us an opportunity to study some processes of astrophysical importance in space. This opportunity has been particularly useful in investigating the photon content in the EHE cosmic ray spectrum. The processes of magnetic pair creation and photon splitting are the basic mechanisms taken into account in our Monte Carlo simulation code. Such processes have been simulated for primary photons in the magnetic field near the Sun to study the characteristics of the cascading of these extraordinary showers. Upon simulation, such cascading particles produced by primary photons with an energy $\geq 10^{19} \mathrm{eV}$ could be detected on the Earth's surface within a solid angle of $6.12 \times 10^{-4}$ sr from the Sun's position. The characteristics of cascading initiated by photons in such a strong magnetic field near the Sun are discussed.
\end{abstract}

Key words. Sun: general - ISM: cosmic rays

\section{Introduction}

The paths of charged particle cosmic rays through the cosmos are deflected by galactic and extragalactic magnetic fields, making it difficult to identify source directions. The integral flux of cosmic rays with primary energy above $10^{19} \mathrm{eV}$ is about one particle per square kilometer per year, probably dropping by a factor of a hundred at energies above $10^{20} \mathrm{eV}$. Although there is evidence that the highest energy particles are predominantly protons (Bird 1993), the number of photons at energies above $10^{19} \mathrm{eV}$ may be significant for two reasons. The EHE cosmic ray protons, distributed uniformly in the universe, produce many photons in collisions with the microwave background radiation photons (Wdowczyk 1990; Halzen 1990). The EHE photons can be also efficiently produced from the decay of massive particles (e.g. Higgs and gauge bosons) as predicted by some exotic theories (Bhattacharjee 1999). Interestingly, this last model predicts that a new component in the cosmic ray spectrum should emerge in an energy region above $\sim 10^{20} \mathrm{eV}$, composed mainly of photons and neutrinos (Bednarek 1999).

Cosmic rays may interact with magnetic fields and lose their energies via the synchrotron process. Electrons produce synchrotron radiation in many astrophysical environments - indeed this radiation is the basis of radioastronomy. At very high particle energies, photons will also lose

Send offprint requests to: A. Mahrous,

e-mail: ayman@crsgm1.crinoue.phy.saitama-u.ac.jp energy in this way, especially in strong magnetic field regions. The propagation of EHE cosmic rays through the galactic magnetic field has been studied by many authors. Hillas (1984) indicated that at energies above $7 \times 10^{19} \mathrm{eV}$, protons will successfully transverse a Milky Way field of $2 \mu \mathrm{G}$ (in the plane of the galaxy and its halo) with little deflection, although this will not be the case for more highly charged cosmic rays like oxygen and iron nuclei. Given the assumption that primary photons are the source of the observed highest energy showers, photons can interact with the Sun's magnetic field and cascading occurs. The solar magnetic field depends on the state or activity of the Sun. The main periodicity in the Sun's activity is the 11-year cycle called the solar cycle. During that cycle, changes occur in the Sun's internal magnetic field and in the surface disturbance level. This cycle is sometimes referred to as the sunspot cycle. Sunspots are manifestations of magnetically disturbed conditions at the Sun's visible surface. At the beginning of a cycle or "solar minimum", the solar magnetic field resembles a dipole whose axis is aligned with the Sun's rotation axis. In this configuration the helmet streamers form a continuous belt about the Sun's equator and coronal holes are found near the poles. During the following 5-6 years towards the "solar maximum", this configuration is totally destroyed, leaving the Sun, magnetically, in a disorganized state with streamers and holes scattered all over different latitudes. During the latter part of the cycle the dipole field is restored. 
In our paper, we studied the development of photontriggered electromagnetic cascades in the Sun's magnetic field during solar minimum, since it is difficult to predict this field during the higher states of solar activities.

\section{Interaction processes}

High energy photon exceeding $10 \mathrm{TeV}$ move along curved magnetic field lines and can convert into an electronpositron $\left(\mathrm{e}^{ \pm}\right)$pair (Anguelov 1999), which is valid for the $\sim 10^{12} \mathrm{G}$ magnetic fields present in pulsar polar caps. The subsequent quantized synchrotron radiation by $\mathrm{e}^{ \pm}$pairs will convert into a second generation of pairs and then an electromagnetic cascade develops in the pulsar magnetosphere. In the present paper, we inserted such mechanisms (magnetic pair creation and photon splitting) in our Monte Carlo simulation code.

\subsection{Pair creation}

In quantum electrodynamics, the process of pair creation of photons takes place in an external magnetic field which can absorb momentum perpendicular to its strength B (Harding 1997). The rate of pair creation (Toll 1952; Klepikov 1954) increases rapidly with increasing photon energy and transverse magnetic field strength, becoming significant for fields approaching the critical field strength of $B_{\mathrm{cr}} \approx 4.414 \times 10^{13} \mathrm{G}$. Assuming EHE photons emitted at very small angles to the magnetic field (Sturrock 1971; Ruderman 1975), such photons will convert into pairs only after traveling a distance $\delta$ comparable to the field line radius of curvature $\rho$, so that $\sin \theta \sim \delta / \rho$ where $\theta$ is the angle between the photon momentum and magnetic field vectors. The above results agree numerically with the analytical expression of the one-photon pair creation rate near threshold energy derived by Baring (1988) for a magnetic field intensity nearly equal or exceeding the value of $0.1 B_{\mathrm{cr}}$. The rate of pair production of an EHE photon with energy $E_{\gamma}$ can be expressed in terms of the attenuation coefficient (Klepikov 1954; Tsai 1974) as:

$\tau_{\mathrm{p}} \approx \frac{\alpha B A_{\mathrm{p}, \mathrm{n}}(\chi) \sin \theta}{2 \lambda B_{\mathrm{cr}}}$

knowing that;

$\chi=\frac{B E_{\gamma} \sin \theta}{2 B_{\mathrm{cr}}}$

where $\alpha=\left(\frac{1}{137}\right)$ and $\lambda=\frac{\hbar}{m_{\mathrm{e}} c}$ (Compton wavelength of the electron), $m_{\mathrm{e}}$ is the electron mass. The labels $\mathrm{p}$, $\mathrm{n}$ of the parameter $A_{\mathrm{p}, \mathrm{n}}(\chi)$ refer to the state in which the photon's electric field vector is parallel and normal to the plane (containing the magnetic field and the photon's momentum vector) respectively. The value of that parameter $A_{\mathrm{p}, \mathrm{n}}(\chi)=(0.31,0.15) \mathrm{e}^{\frac{-4}{3 \chi}}$ for $\chi \ll 1$, and $A_{\mathrm{p}, \mathrm{n}}(\chi)=(0.72,0.48) \mathrm{e}^{\frac{-1}{3}}$ for $\chi \gg 1$.

\subsection{Photon splitting}

The probability of pair creation and photon splitting for an EHE photon with energy $E_{\gamma}$ in a magnetic field of strength $B$ increases when $B$ is at least a significant fraction of the quantum critical field $B_{\mathrm{cr}}$. The splitting rate of photon in external magnetic field is expressible (Papanyan 1972) in terms of the splitting attenuation coefficient as:

$\tau_{\mathrm{s}} \approx \frac{k \alpha^{3} B^{6} \varepsilon(B) E_{\gamma}^{5}(\sin \theta)^{6}}{10 \lambda \pi^{2} B_{\mathrm{cr}}^{6}}$

where $k=\left(\frac{19}{315}\right)^{2}, \varepsilon(B)=1$ for $B \ll B_{\text {cr }}$ and equal $B^{-6}$ for $B \gg B_{\mathrm{cr}}$. We simulated such processes using the Monte Carlo method, and Erber's rates (Erber 1966) were used to get the exact quantum mechanical rates for $\mathrm{e}^{ \pm}$pair creation by photons and photon splitting by secondary $\mathrm{e}^{ \pm}$pairs.

\subsection{Probability of interaction}

The total probabilities per unit length $(1 \mathrm{~cm})$ for pair creation and photon splitting are given by (Anguelov 1999)

$\psi=\frac{q}{E_{\mathrm{e}}^{\frac{1}{3}}} \int_{0}^{1}\left(\frac{(1-u)^{\frac{5}{3}}}{u^{\frac{2}{3}}}+\frac{1}{(1-u)^{\frac{1}{3}} u^{\frac{2}{3}}}\right) \mathrm{d} u$

where $q=3.9 \times 10^{6}\left(\frac{B}{B_{\mathrm{cr}}}\right)^{\frac{2}{3}} \frac{\mathrm{GeV}^{\frac{1}{3}}}{\mathrm{~cm}}, u=\frac{E_{\gamma}}{E_{\mathrm{e}}-E \gamma}, E_{\gamma}$ and $E_{\mathrm{e}}$ are the energies of photon and electron respectively. The above equation can be used to sample the energies of the secondary particles such as photons in photon splitting and electrons in pair creation.

\section{The Sun's magnetic field}

The interplanetary magnetic field of the Sun is formed as a result of the transport of the photospheric magnetic field (Amenomori 2000) by the solar wind flowing continuously from the Sun (Parker 1963). Field lines near the solar equator form closed loops (neutral sheets), while field lines from the poles are dragged far into interplanetary space by the high-speed solar wind. Furthermore, the magnetic field of the Sun changes sign from south to north across the neutral sheets. The interplanetary magnetic field can be explained well by the Ballerina Skirt model assuming a rotating dipole in the Sun (Schultz 1973; Saito 1975; Svalgaard 1978), although direct evidence for the presence of such a rotating dipole has not yet been obtained. During the solar maximum, a disturbance occurred in the solar magnetic field due to the influence of active regions on the Sun's surface. That field can dominate at mid to low solar latitudes (in the solar chromosphere) by up to 2 orders of magnitude over the dipole component near the Sun, knowing that the magnetic dipole moment of the Sun equals $6.87 \times 10^{32} \mathrm{G} \mathrm{cm}^{3}$. 


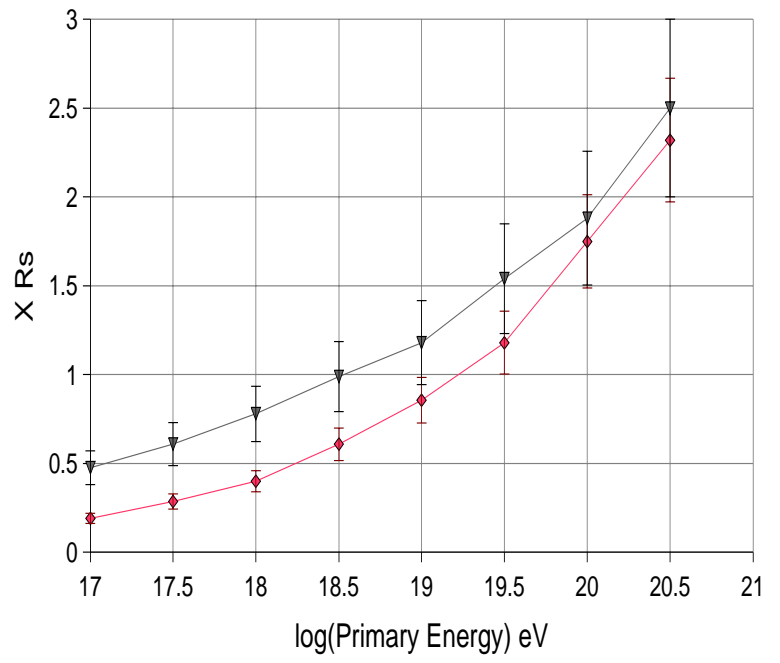

Fig. 1. The first interaction point for different energies of the incident primary photons injected $\|$ (upper curve) and $\perp$ (lower curve) to the SMEP at different locations from the Sun's surface.

\section{Simulation results}

We injected photons randomly with different energies in two directions (parallel $\|$ and perpendicular $\perp$ to the Solar Magnetic Equatorial Plane (SMEP)) within a circle of radius $n R_{\mathrm{S}}$ around the Sun, (where $\mathrm{n}$ is an integer number ranging from 0 to 3 , and $R_{\mathrm{s}}$ is the Sun's radius). Magnetic cascading initiated by primary monoenergetic photons with energies between $10^{17}$ and $10^{20.5} \mathrm{eV}$ was simulated. The primary energies of ten simulated photons are assigned with a step of $(\Delta \log E=0.5 \mathrm{eV})$ over the energy range, and averaged over 10 simulations. Our simulation shows that the probability of interaction depends not only on the primary photon energy but also on the angle of incidence. Figure 1 shows the first interaction point (measured from the Sun's surface) as a function of the energy of the incident primary photons injected into the two directions mentioned before. The figure also shows that $\perp$ showers (lower curve) start cascading at much deeper positions than || showers (upper curve) to the SMEP. For the highest energy \| showers with energy of $10^{20.5} \mathrm{eV}$, cascading starts at a distance of $2.5 R_{\mathrm{s}}$ from the Sun's surface; this value is reduced to $2.3 R_{\mathrm{s}}$ in a case of $\perp$ showers. The difference between the two curves becomes much wider $\left(\approx 0.3 R_{\mathrm{s}}\right)$ for the lowest energy showers with primary energy $10^{17} \mathrm{eV}$ which cascade very near to the Sun at distance $0.5 R_{\mathrm{S}}$ from the Sun's surface. In addition, fluctuation is much larger for the higher energy region. We studied the characteristics of magnetic cascading development for $\perp$ showers (Fig. 2) and $\|$ showers (Fig. 3) initiated by EHE photons. The total number of generated photons cascading along the path up to a given altitude from the Sun's surface (with step of $2 \times 10^{4} \mathrm{~km}$ ) has been averaged and plotted for the primary photons with energies (curves from top to bottom) $10^{20.5}, 10^{20}, 10^{19.5}, 10^{19}, 10^{18.5}, 10^{18}$, $10^{17.5}$ and $10^{17} \mathrm{eV}$. The comparison between the two figures shows that the number of photons generated is much

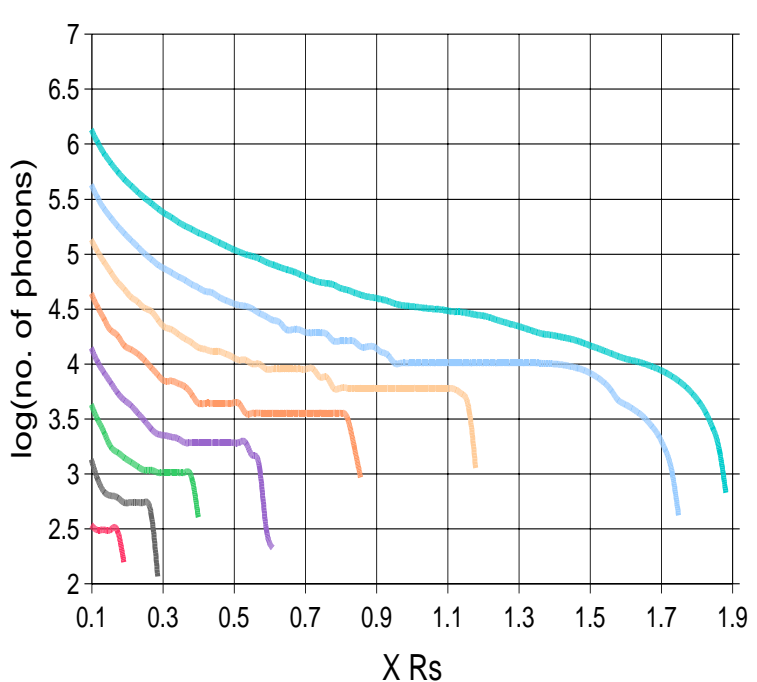

Fig. 2. Magnetic cascading development for $\perp$ showers initiated by EHE photons with energies (curves up to down) $10^{20.5}$, $10^{20}, 10^{19.5}, 10^{19}, 10^{18.5}, 10^{18}, 10^{17.5}$ and $10^{17} \mathrm{eV}$.

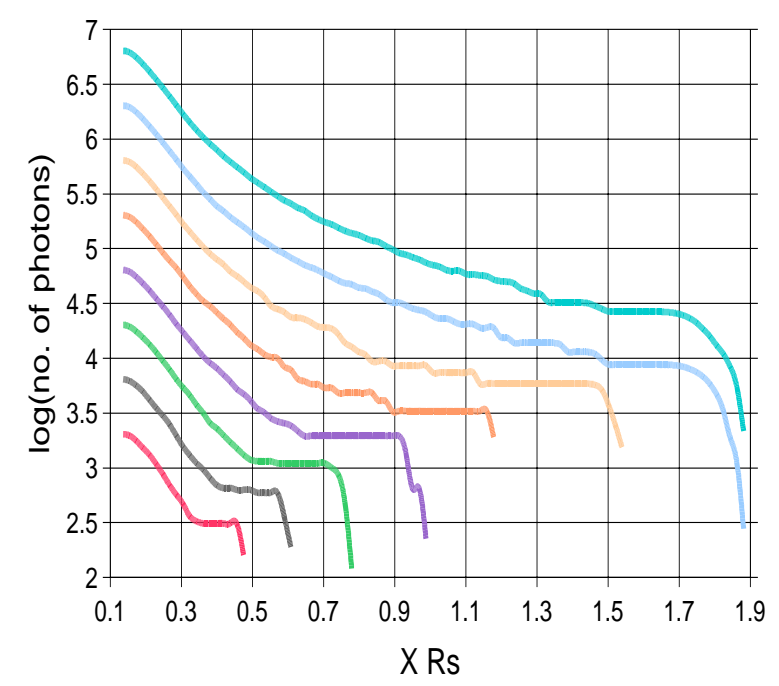

Fig. 3. Magnetic cascading development for $\|$ showers initiated by EHE photons with energies (curves up to down) $10^{20.5}, 10^{20}$, $10^{19.5}, 10^{19}, 10^{18.5}, 10^{18}, 10^{17.5}$ and $10^{17} \mathrm{eV}$.

higher in case of $\|$ showers than $\perp$ showers. We calculated the integrated photon energy spectra of the total number of secondary photons generated along the path up to the Sun's surface. These results have been plotted for $\perp$ and $\|$ showers with step of $(\Delta \log E=0.1)$ and primary energies (curves top to bottom) $10^{20}, 10^{19.5}, 10^{19}, 10^{18.5}$, $10^{18}, 10^{17.5}$ and $10^{17} \mathrm{eV}$ as shown in Figs. 4 and 5 respectively. Due to computer (CPU) time limitation, the spectral analysis of the generated secondary photons is processed for energies up to $10^{4} \mathrm{GeV}$ for $\perp$ showers and $10^{5.4} \mathrm{GeV}$ for $\|$ showers. The number of highest energy photons generated is greater in $\|$ showers than $\perp$ showers, which is compatible with the characteristics of the longitudinal development curves (see Figs. 2 and 3). The energy weighted-spectrum for $\perp$ and $\|$ showers within the same energy levels listed before are shown in Figs. 6 and 7 respectively. The two figures show that the energy-weighted 


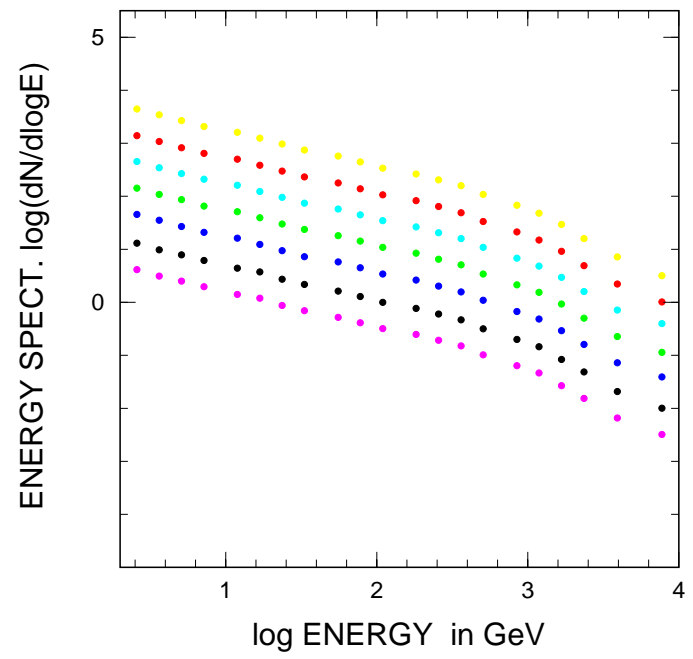

Fig. 4. Integrated photon energy spectra for $\perp$ showers initiated by EHE photons with energies (curves up to down) $10^{20}$, $10^{19.5}, 10^{19}, 10^{18.5}, 10^{18}, 10^{17.5}$ and $10^{17} \mathrm{eV}$.

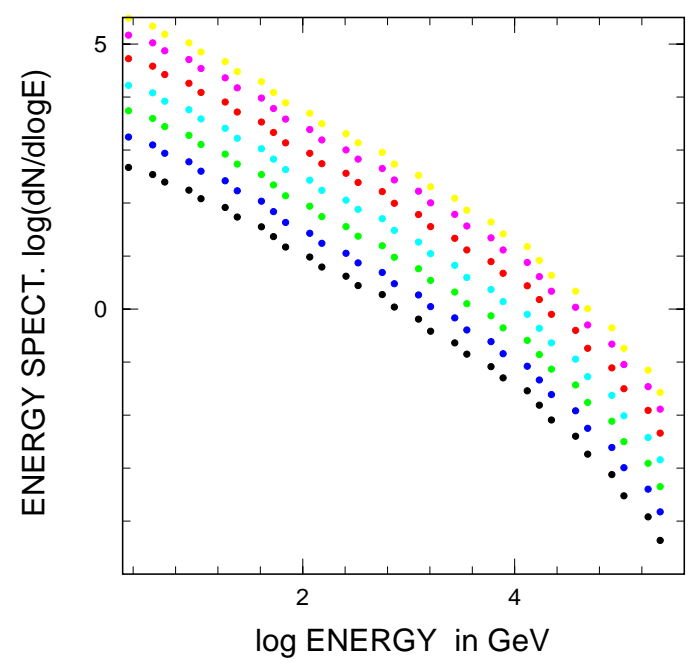

Fig. 5. Integrated photon energy spectra for $\|$ showers initiated by EHE photons with energies (curves up to down) $10^{20}$, $10^{19.5}, 10^{19}, 10^{18.5}, 10^{18}, 10^{17.5}$ and $10^{17} \mathrm{eV}$.

spectrum curves for $\perp$ has a maximum at a photon energy of $10^{3.5} \mathrm{GeV}$, while this maximum appears at a photon energy of $10^{4.7} \mathrm{GeV}$ in the case of $\|$ showers. These can be referred to the distribution of the highest energy particles generated through the photon energy spectra. The electron component of the cascading is characterized by large fluctuations and is not significant in the cascading, therefore, the electron component is not mentioned in our results which rather concentrate on the photon content of the cascading.

\section{Discussions}

Our simulation shows that $\perp$ showers cascade much deeper than || showers to the SMEP. This could be explained by $\perp$ showers having impact parameters larger than the solar radius, and so they propagate parallel to the magnetic

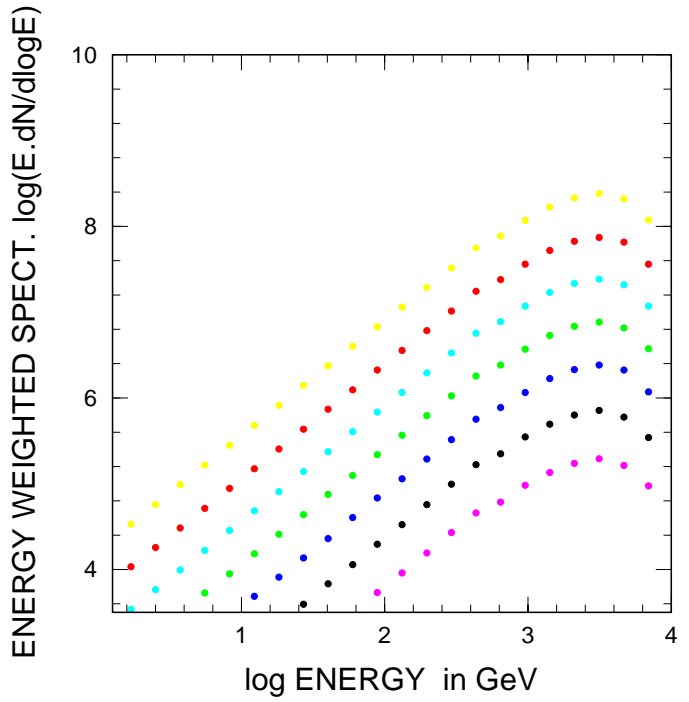

Fig. 6. The energy weighted spectrum for $\perp$ showers initiated by EHE photons with energies (curves up to down) $10^{20}, 10^{19.5}$, $10^{19}, 10^{18.5}, 10^{18}, 10^{17.5}$ and $10^{17} \mathrm{eV}$.

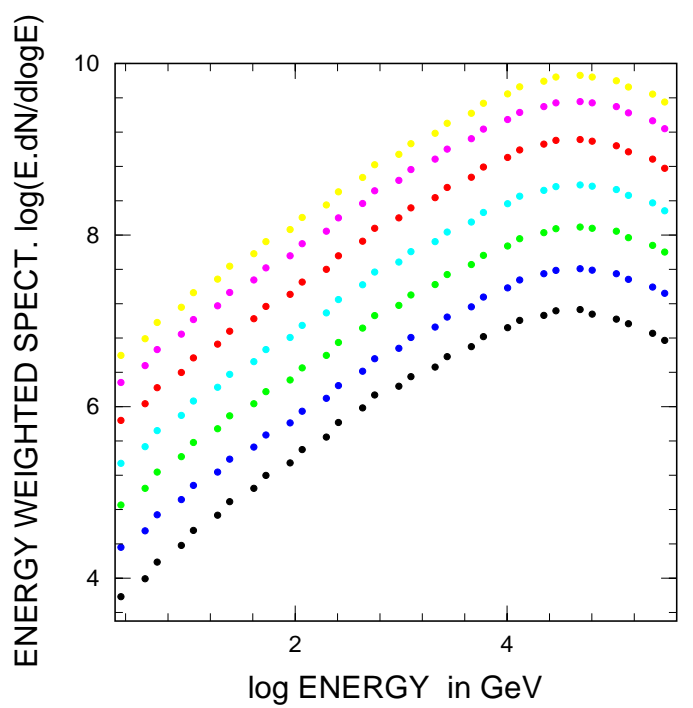

Fig. 7. The energy weighted spectrum for $\|$ showers initiated by EHE photons with energies (curves up to down) $10^{20}, 10^{19.5}$, $10^{19}, 10^{18.5}, 10^{18}, 10^{17.5}$ and $10^{17} \mathrm{eV}$.

dipole, far away from the magnetic poles. On the other hand, a substantial fraction of $\|$ showers should perpendicularly cross the Sun's polar magnetic field which increases their probability of interaction. The simulation also shows that EHE gamma-rays with energies between $10^{19}$ and $10^{20.5} \mathrm{eV}$ start cascading in the Sun's magnetic field within a circle of radius $2 \sim 3$ times the radius of the Sun. If we are trying to detect such cascading particles on the Earth with air shower arrays, these showers should be detected within a solid angle equal $\Delta \Omega \approx 6.12 \times 10^{-4}$ sr from the Sun's position. The question arising now is how to distinguish such unusual showers from ordinary showers. In the case of photons expected in the cosmic ray spectrum above $10^{19} \mathrm{eV}$, the answer is simply that secondary photons of the cascades produced in the Sun's magnetosphere arrive 


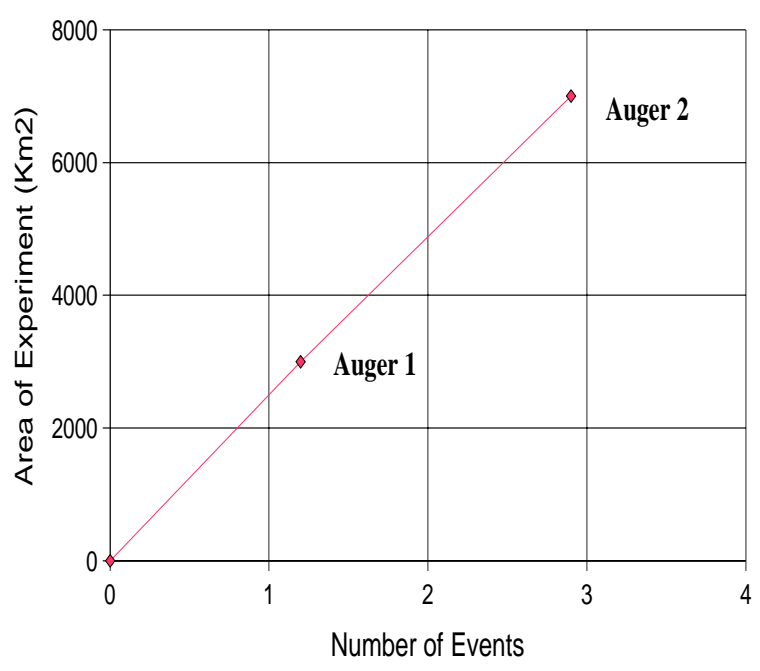

Fig. 8. The expected rate of cascading showers in the Sun's magnetic field detected by Auger 1 and 2 experiments.

at the Earth's atmosphere with a significant perpendicular extent which is the result of deflection of the paths of secondary electrons by the Sun's magnetic field. This could be a characteristic phenomenon of such unusual showers. In addition, the radius of the curvature and deflection angle of such cascading showers in the Sun's magnetic field can be simulated at the top of the Earth's atmosphere, which will be presented in future work. It could be helpful to estimate the expected rate of such showers detected by the largest ground array experiments, such as the Auger experiment in Argentina. Assuming the Auger location, the number of these showers detected from a circle of $2 R_{\mathrm{s}}$ around the Sun is given by:

$Z=S \Delta \Omega N$

where $S$ is the part of the sky observed by the experiment, $N$ is the total number of observed showers. Figure 8 shows the expected rate per 10 years for such showers to be detected by the planned experiments Auger 1 and 2 with total areas of $3000 \mathrm{~km}^{2}$ and $7000 \mathrm{~km}^{2}$ respectively. Although the predicted rate of such showers is very small (about 1.2 and 3 showers per 10 years for Auger1 and 2), it is possible to enlarge the detecting area by extending the detector spacing up to $3 \mathrm{~km}$ to observe this kind of EHE shower in the future.

Acknowledgements. The authors would like to thank Prof. H. Vankov for his useful comments and discussions.

\section{References}

Amenomori, M., et al. 2000, Phys. Rep. [astro-ph/0008159] Anguelov, V., \& Vankov, H. 1999, J. Phys. G: Nucl. Part. Physics, 251, 10

Baring, M. G. 1988, MNRAS, 235, 79

Bednarek, W. 1999, Astro Phys. [astro-ph/9911266]

Bhattacharjee, P., \& Sigl 1999, Phys. Rep. [astro-ph/9811011]

Bird, D. J., Corbató, S. C., Dai, H. Y., et al. 1993, Phys. Rev. Lett., 71, 3401

Erber, T. 1966, Rev. Mod. Phys., 38, 626

Halzen, F., Protheroe, R. J., Stanev, T., Vankov, H. P., et al. 1990, Phys. Rev. D, 41, 342

Hillas, A. M. 1984, ARA, 22, 425

Harding, A. K., \& Baring, M. G. 1997, ApJ, 476, 246

Klepikov, N. V. 1954, Zh. Eksp. Theor. Fiz., 26, 19, first citation in article

Papanyan, V. O., \& Ritus, V. I. 1972, Sov. Phys., 34, 1195

Parker, E. J. 1963, Interplanetary Dynamical Process (New York: Interscience)

Ruderman, M. A., \& Sutherland, P. G. 1975, ApJ, 196, 51

Saito, T. 1975, Sci. Rep. Tohoku Univ., Ser., 5, 23, 37

Schultz, M. 1973, Astrophys. Space Sci., 24, 371

Sturrock, P. A. 1971, ApJ, 164, 529

Svalgaard, L., \& Wilcox, J. M. 1978, ARA\&A, 16, 429

Toll, J. S. 1952, Ph.D. Thesis, Princeton Univ., first citation in article

Tsai, W. Y., \& Erber, T. 1974, Phys. Rev. D, 10, 492

Wdowczyk, J., \& Wolfendale, A. W. 1990, ApJ, 349, 35 